\title{
The Analytical Transfer Matrix Method Combined with Supersymmetry: Coulomb Potential
}

\author{
Hosung Sun

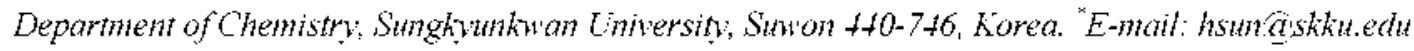 \\ Recerved November 20,2006
}

\begin{abstract}
Combining the analytical transfer matrix method with supersyymetry algebra, a new quantization condition is suggested. To demonstrate the efficiency of the new quantization condition, the eigenenergies of the Coulomb potential are analytically derived. The scattering-led phase shifts are also determined and they are the same for all Coulomb potential states. It is found that the new quantization condition is mathematically simple and exact.
\end{abstract}

Key Words : Coulomb potential. ATMM. Supersymmetry

\section{Introduction}

For a one-dimensional șystem. when a particle is moving between two classical turning points. its kinetic energy: cannot be larger than the potential energy. Therefore the particle cannot escape out from the potential well but oscillates between the two turning points. However quantum mechanically the particle can enter the classically forbidden region. The exponentially decaying wave function in the classically forbidden region should be connected smoothly to the oscillating wave function in the classically allowed region at the classical turning points. The wave reflected at the turning point has a different phase. relative to the incoming wave traveling towards the turning point. Therefore the phase of wave function should change through the turning points and the change is called a phase shift. Furthermore. there exists a scattering phenomenon between the two turning points unless the potential is constant. The scattering also causes an additional phase shift that is called the scattering-led phase shift. ${ }^{\text {l }}$

The existence of the additional phase shift is ignored in the well known WKB (Wentzel-Kramers-Brillouin) approximation (or quantization condition.). ${ }^{23}$ This is the reason why the WKB quantization is not exact though it is found to be exact for the harmonic oscillator potential and the Morse potential. The recently developed analytical transfer matrix method (ATMM) suggests a new quantization condition that explicitly includes the additional phase shift. The so-called complete quantum momentum function is suggested and the integration of the quantum momentum function over position variable yields the quantization condition that can describe the motion of a particle oscillating within a classically bound region. The ATMM quantization condition has been applied to various potentials and is considered to be exact. ${ }^{+-\delta}$

We utilize the ATMM quantization condition to generate the eigenenergy and the additional phase shift of the Coulomb potential. This approach enables one to directly evaluate the phase shift. However. there is a great difficulty in evaluating the phase shift because the phase shift is expressed in terms of eigenfunctions. That is one has to predetermine the eigenfunctions of all eigenstates to use the ATMM quanti- zation. For the one-dimensional Coulomb potential, the eigenfunctions are of course. known. Even so. the mathematical evaluation of the phase shift is still very difficult because the eigenfunctions of excited states are very complicated. ${ }^{9}$

In this work. adopting the supersymmetry algebra. a new and simple quantization condition for evaluating the phase shift and eigenenergy is suggested. ${ }^{10-12}$ Since the supersymmetric partner Hamiltonians share the conmon eigenenergies of the original Hamiltonian. only the ground state eigenfunctions (or superpotentials) of partner Hamiltonians. instead of all the eigenfunctions of the original Hamiltonian. are required to evaluate the phase shift when the new quantization condition is used. It reduces a mathematical difficulty a lot.

In the following sections the Coulomb potential system is explicitly defined. The supersymmetry and the ATMM quantization condition are introduced. Particularly the superșinmetry adopted version of the ATMM quantization condition is presented. The eigenenergy and the phase shift of the Coulomb potential are presented and analyzed. Finally the information obtained from this work is provided in the conclusion section.

\section{Coulomb Potential}

The Coulomb potential energy function is widely used in chemistry. ${ }^{13}$ And the most well known one is the protonelectron attraction potential in a hydrogen atom. The onedimensional Coulomb potential problem can be analytically solved. The eigenfunctions of the Coulomb potential are expressed in termı of the Laguerre polynomials and the eigenenergies are obtained in a closed form. Let us define the one-dimensional Coulomb potential explicitly by taking a simple example.

The radial Schrödinger equation for a hydrogenlike atom containing one electron of mass $M_{e}$ and a nucleus of mass $M$. for a certain angular momentum quantum number $l$ is

$$
\begin{aligned}
& {\left[-\frac{\hbar^{2}}{2 \mu} \frac{d^{2}}{d r^{2}}+\frac{\hbar^{2}}{2 \mu} \frac{l(l+1)}{r^{2}}-\frac{Z e^{2}}{r}\right] \Psi_{N l}(r)=E_{X} \Psi_{W}(r)} \\
& (0<r<\infty)
\end{aligned}
$$


where $\mu=\left[M_{i} /\left(1+M_{i} / M\right)\right]$ is the reduced mass, $Z e$ is the nuclear charge and. of course. $e$ is the unit charge. The attraction potential between the electron and the nucleus $-\left(Z e^{2} / r\right)$ is zero at $r \rightarrow \infty$. The Schrödinger equation Eq. (1) can be exactly solved to generate the $N$-dependent eigenenergy $E_{N}$ where

$$
E_{N}=-\frac{\mu Z^{2} e^{+}}{2 \hbar^{2} N^{2}} \quad N=1.2 .3 \ldots
$$

The $N$ is called the principal quantum number. The wave function (or eigenfunction) $\Psi_{W}(r)$ depends not only on $I$ but also on $N . \Psi_{N /}(r)$ is equivalent to $r R_{M}(r)$ where $R_{M}(r)$ is the customarily defined radial function of a hydrogenlike atom. ${ }^{13}$ The boundary condition is that $R_{\mathrm{v}}(r)$ goes to zero at infinity: Now the angular momentum quantum number $l$ is limited as $l=0.1,2, \ldots, N-1$ in order to satisfy the boundary condition.

The Schrödinger equation Eq. (1) can be rewritten as

$$
\left[-\frac{d^{2}}{d r^{2}}+\frac{l(I+1)}{r^{2}}-\frac{q^{2}}{r}\right] \Psi_{N / 2}(r)=\varepsilon_{N} \Psi_{N 2}(r) \quad(0<r<\infty)
$$

where $q^{2}=\frac{2 \mu Z e^{2}}{\hbar^{2}}$ and $\varepsilon_{N}=\frac{2 \mu}{\hbar^{2}} E_{N}=-\frac{q^{4}}{4 N^{2}}$.

For convenience a new quantum number $n=N-I-1(n=$ $0,1,2, \ldots)$ is introduced. $n$ is. in nature, equivalent to the number of nodes in the radial wave function $\Psi_{N I}(r)$.

In summary. the Coulomb potential sy stem of a certain $/$ is as follows: $:^{13-15}$

$$
H \Psi_{n}(r) \equiv\left[-\frac{d^{2}}{d r^{2}}+V(r)\right] \Psi_{n}(r)=\varepsilon_{n} \Psi_{n}(r) \quad(0<r<\infty)
$$

where the Coulomb potential $V(r)=-\frac{q^{2}}{r}+\frac{I(l+1)}{r^{2}}$

and the eigenenergy $\varepsilon_{n}=-\frac{q^{+}}{4(n+l+1)^{2}}$ for $n=0,1,2$.

Eqs. (4) and (5) are the Coulomb potential system whose eigenenergy' and phase shift will be evaluated using the ATMM quantization condition ${ }^{1}$ with the aid of supersymumetry. ${ }^{10.12}$ In the next section the supersymmetry is briefly reviewed and a new ATMM quantization condition combined with supersymmetry is introduced

\section{ATMM Combined with Supersymmetry}

First some characteristics of supersymunetry are briefly presented. According to the supersymmetric quantun mechanics. ${ }^{12}$ the raising and lowering operators $A_{=}^{(s)}$ for the $s$-th supersymmetric partner Hamiltonian $H^{(s)}$ are defined as

$$
A_{=}^{(s)}=\mp \frac{d}{d r}+W^{(s)}(r) \quad s=0,1,2, \ldots
$$

The superscript $(s)$ is the hierarchy index so that it indicates the $s$-th partner Hamiltonian. In this work, the $s=0$ case is the original Hamiltonian. For example, $H^{i j 1}$ is equivalent to the original $H$ in $\mathrm{Eq}$. (4). Likewise. $V^{(i)}(r)=V(r)$, $\Psi_{n}^{(i)}(r)=\Psi_{n}(r)$. and $\varepsilon_{n}^{(1)}=\varepsilon_{n}$. Here the superpotential $W^{(s)}(r)$ is. in essence. the minus log derivative of the ground state $(n=0)$ wave function $\Psi_{i 1}^{(s)}(r)$ of Hamiltonian $H^{(s)}$. i.e.

$$
W^{(s)}(r)=-\frac{d \Psi_{i j}^{(s i}(r) / d r}{\Psi_{\varphi !}^{(s)}(r)}
$$

And it satisfies a following Riccati equation

$$
\frac{d W^{(s)}(r)}{d r}=\left[W^{(s)}(r)\right]^{2}-V^{(s)}(r)+\varepsilon_{\mathrm{l}}^{(s)} .
$$

Then the original Hamiltonian in Schrödinger equation Eq. (4) can be rewritten as

$$
\begin{aligned}
H^{(j)}(=H) & =-\frac{d^{2}}{d r^{2}}+V^{(i)}(r)=A_{-}^{(j)} A_{-}^{(j)}+\varepsilon_{i j}^{(j)} \\
& =-\frac{d^{2}}{d r^{2}}+\left[W^{(i)}(r)\right]^{2}-\frac{d W^{(i)}(r)}{d r}+\varepsilon_{i j}^{(0)} .
\end{aligned}
$$

The first supersymmetric partner Hamiltonian is

$$
\begin{aligned}
H^{(1)} & =A_{-}^{(1)} A_{-}^{(())}+\varepsilon_{0 !}^{(i)}=A_{+}^{(1)} A_{-}^{(1)}+\varepsilon_{0}^{(1)} \\
& =-\frac{d^{2}}{d r^{2}}+\left[W^{(1)}(r)\right]^{2}-\frac{d W^{(1)}(r)}{d r}+\varepsilon_{0}^{(1)} .
\end{aligned}
$$

In general the partner Hamiltonian can be generated for any index s.i.e.

$$
\begin{aligned}
H^{(s)} & =A_{+}^{(s)} A_{-}^{i s)}+\varepsilon_{01}^{(s)} \\
& =-\frac{d^{2}}{d r^{2}}+\left[W^{(s)}(r)\right]^{2}-\frac{d W^{(s)}(r)}{d r}+\varepsilon_{0 i}^{(s)} .
\end{aligned}
$$

From the supersynmetry algebra we easily obtain the following relations, ${ }^{12}$

$$
\begin{gathered}
\varepsilon_{s}=\varepsilon_{i}^{(s)} \\
\Psi_{s}(r)=A_{+}^{(s)} A_{-}^{(s-1)} \cdots A_{-}^{(1)} \Psi_{i j}^{j s !}(r) \\
\Psi_{i j}^{(s)}(r)=C^{(s)} \exp \left[-\int_{r} W^{(s)}(t) d t\right]
\end{gathered}
$$

where $C^{(s)}$ is a normalization constant. Note that $\Psi_{s}(r)$ is the $s$-th state $(n=s)$ wave function of the Coulomb potential Hamiltonian $(H)$ and $\Psi_{41}^{(s)}(r)$ is the ground state $(n=0)$ wave function of $H^{(s)}$. Consequenty w'e find that the hierarch index sis equivalent to the quantum number $n$ of the original Hamiltonian. Therefore on we can safely use the notation of $n$ in replacement of $s$. In Figure 1. the hierarchy of supersynmetric partner Hamiltonians. Eq. (13) is illustrated. A similar illustration for a rigid symmetric top rotor can be found in this journal. ${ }^{16}$

Now let us introduce the quantization condition of the analytical transfer matrix method. ${ }^{1}$ The ATMM quantization condition. for the Scluödinger equation $\mathrm{Eq}$. (4). can be 

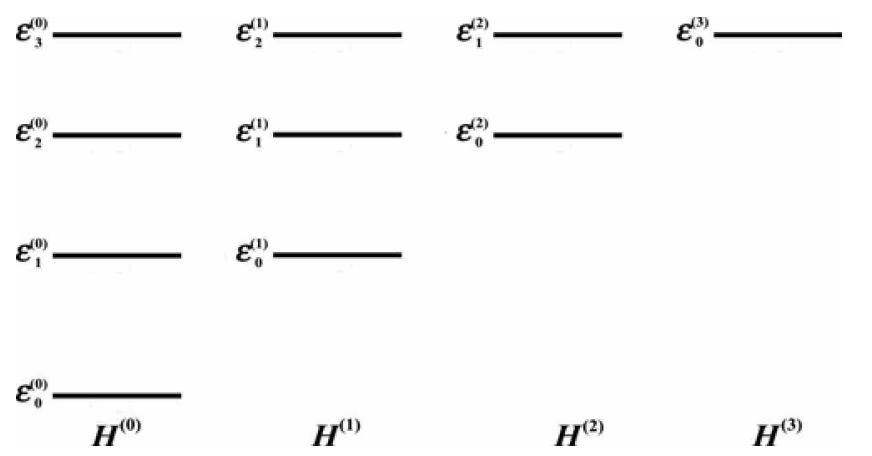

Figure 1. Hierarchy of supersymmetric partner Hamiltonians.

sunumarized as

$$
\int_{r_{L}}^{r_{R}} \kappa_{n}(r) d r+\delta_{n}=(n+1) \pi \quad n=0.1 .2, \ldots
$$

where $n$ is the quantum number. $\kappa_{n}$ is the momentum. i.e.

$$
\kappa_{n}^{-}(r)=\sqrt{\varepsilon_{n}-V(r)}
$$

where $V(r)$ is the potential and $\varepsilon_{n}$ is the eigenenergy of the $n$ th state. $r_{L}$ and $r_{R}$ are inner (short distance) and outer (long distance) turning point. respectively, i.e. $V\left(r_{L}\right)=V\left(r_{R}\right)=$ $\varepsilon_{\text {i }}$ and $r_{L}<r_{R} . \kappa_{n}(r)$ is always positive between $r_{L}$ and $r_{R}$.

The so-called scattering-led phase shift $\delta_{i}$ is

$$
\delta_{n}=-\int_{r_{L}}^{r_{R}} \kappa_{n}^{\prime}(\mu) \frac{P_{n}(r)}{P_{n}^{\prime}(r)} d r
$$

where $\kappa_{n}^{\prime}(r)=\frac{d \kappa_{n t}(r)}{d r^{r}}$ and $P_{n}^{\prime}(r)=\frac{d P_{n}(r)}{d r} . P_{n}(r)$ is the minus $\log$ derivative of the $n$-th state wave function $\Psi_{n}(r)$. i.e.. $P_{n}(r)=-\frac{d \Psi_{n}(r) / d r}{\Psi_{n}(r)}$. Note that $P_{0}(r)=W(r)$.

In order to evaluate the phase shift $\delta_{\mathrm{ft}}$ in Eq. (18) one must predetermine the wave function $\Psi_{n}(r)$ of $H\left(=H^{(i)}\right)$. which requires a lot of algebra. Even the $\Psi_{n}(r)$ is known. the integration is very difficult because of the complexity of the wave function. Here we adopt the supersynmetry algebra to avoid this difficulty. The quantization condition, $\mathrm{Eq}$. (16) should be valid not only for $H$ (or $\Psi_{n}(r)$ ) but also for any partner Hamiltonian $H^{(n)}$ (or $\left.\Psi_{01}^{(n i}(r)\right)$. $\Psi_{n}(r)$ and $\Psi_{01}^{(n)}(r)$ are of course different to each other but the eigenenergies are identical, i.e., $\varepsilon_{n}=\varepsilon_{0}$ ) (see Eq. (13).) Therefore one can rewrite the quantization condition only for the ground state $(n=0)$ of any $n$-th supersymmetric partner Hamiltonian $H^{(i)}$. i.e.

$$
\begin{gathered}
\int_{r_{L}^{(n)}}^{r_{R}^{(n)}} K_{0}^{i n !}(r) d r+\delta_{0}^{(n)}=\pi \\
\kappa_{0}^{(n)}(r)=\sqrt{\varepsilon_{0}^{(n !}-V^{(n)}(r)}=\sqrt{\varepsilon_{n}-V^{(n)}(r)} \\
\delta_{0 !}^{(n)}=-\int_{r_{L}^{\prime n !}}^{r_{R}^{(n)}} \frac{d K_{0}^{(n)}(r)}{d r} \frac{W^{(n)}(r)}{d W^{(n)}(r) / d r} d r .
\end{gathered}
$$

The superscript ( $n$ ) denotes the hierarchy of parther Hamiltonians and the subscript number $n$ (or 0 ) represents the $n$-th state (or ground state) of original Hamiltonian. Needless to say these two indices can be used interchangeably. The new quantization condition (Eqs. (19), (20), and (21)) will be applied to the Coulonb potential system.

The difference between the original ATMM (Eq. 16) and the new ATMM with supersymmetry (Eq. 19) lies in what kind of eigenfunctions is used. In the original ATMM all the eigenfunctions (including excited ones) of the original Hamiltonian are required while in the new ATMM only the eigenfucntions of the ground state of partner Hamiltonians are required. It is much easier to evaluate the ground state eigenfunctions than the excited state eigenfunctions. Furthermore the integration involving only the ground state eigenfunctions is also easier.

\section{Eigenenergy and Phase Shift of Coulomb Potential}

The Coulomb potential is introduced earlier. Using the superșymmetry algebra we can determine the superpotential. etc. The direct way of determining $V^{(n)}(r)$ and $W^{(n)}(r)$ is as follows. Solving the Riccati equation. Eq. (9) with the original potential $V^{(0)}(r)$, one obtains $W^{(0)}(r)$ and $\varepsilon_{i j}^{(i)}$. Then using the supersynmmetry relationship ${ }^{12}$ of $V^{(j+1)}(r)=\left[W^{\langle s|}(r)\right]^{2}+\frac{d W^{(r)}(r)}{d r}+\varepsilon_{0 !}^{i s !}$. one can easily detemine $V^{(1)}(r)$ from $W^{(1)}(r){ }^{17}$ With the partner potential $V^{r^{1]}}(r)$, one again solves the Riccati equation to obtain $W^{\prime \prime}(r)$ and so on. This process can be repeatedly performed up to any hierarchy $n$ (or $s$ ). The results for the Coulomb potential Hamiltonian given in Eq. (4) are

$$
\begin{aligned}
V^{\prime n i}(r) & =-\frac{q^{2}}{r}+\frac{(n+1)(n+1+1)}{r^{2}} \\
W^{\prime n !}(r) & =\frac{q^{2}}{2(n+1+1)}-\frac{n+1+1}{r} \\
\varepsilon_{0}^{(n)} & =-\frac{q^{+}}{4(n+1+1)^{2}}=\varepsilon_{n} .
\end{aligned}
$$

Of course, the eigenenergies in Eq. (24) are identical with those in Eq. (6).

Now using the new ATMM with supersynmetry, i.e.. Eqs. (19). (20). and (21), we detemine the eigenenergy of the Coulomb potential Hamiltonian. First the momentum part is

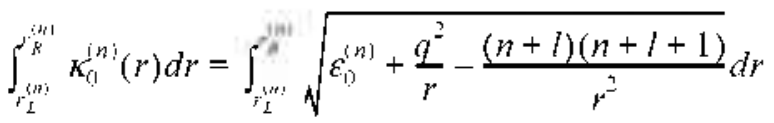

$$
\begin{aligned}
& =\frac{\pi q^{2}}{2}\left(-\varepsilon_{i !}^{i n !}\right){ }^{1 / 2}-\pi(n+l)^{1: 2}(n+1+1)^{1: 2} \text {. }
\end{aligned}
$$

The above integral can be found in a literature. ${ }^{15}$

The phase shift of the $n$-th partner Haniltonian $H^{(n)}, \delta_{0}^{n \text { i }}$ in Eq. (21) can be rewritten as. using Eqs. (22) and (23).

$$
\delta_{0}^{(n)}=-\int_{r_{L}^{(n)}}^{r_{R}^{(n)}} \frac{d}{d r}\left(\sqrt{\varepsilon_{0}^{(n)}+\frac{q^{2}}{r}-\frac{(n+l)(n+l+1)}{r^{2}}}\right)
$$




$$
\times \frac{q^{2} / 2(n+1+1)-(n+1+1) / r}{(n+1+1)^{2} / r^{2}} d r
$$

Let $y=\sqrt{(n+l)(n+l+1)} / r, y_{L}^{(n)}=\sqrt{(n+l)(n+l+1)} / r_{L}^{(m)}$. $y_{R}^{(n)}=\sqrt{(n+l)(n+l+1)} / r_{R}^{(n)}$. then Eq. (26) is reduced to

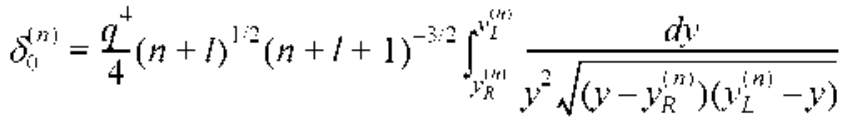

$$
\begin{aligned}
& -\frac{q^{2}}{2}(n+l+1)^{-1}(2 n+2 l+1) \int_{y_{R}^{\prime(y)}}^{y_{L}^{\prime \prime \prime}} \frac{d y}{y^{2} \sqrt{\left(y-y_{R}^{(n)}\right)\left(y_{L}^{(n)}-y\right)}}
\end{aligned}
$$

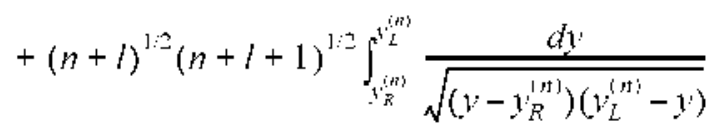

Since $r_{L}^{(n)}$ and $r_{R}^{(n)}$ are the turning points i.e. $V^{(n)}\left(r_{L}^{(n)}\right)=$ $V^{i n j}\left(r_{R}^{(n)}\right)=\varepsilon_{0 !}^{(n)}$ and $0<r_{L}^{(n j}<r_{R}^{i n !}$. one immediately finds that $y_{L}^{(n)}>y_{R}^{(n)}>0$ and the relations; $y_{R}^{(n)}+y_{L}^{(n)}=$ $q^{2} / \sqrt{(n+l)(n+I+1)}$ and $y_{R}^{\prime n)} y_{L}^{(n)}=-\varepsilon_{(i)}^{(n !}$.

Though the above integrals can be found in integration tables. ${ }^{1 /}$ we list the expressions for the convenience of interested readers. For real numbers $b>a>0$.

$$
\begin{aligned}
& \int_{a}^{b} \frac{d y}{\sqrt{(y-a)(b-y)}}=\pi \cdot \int_{a}^{b} \frac{d y}{y \sqrt{(y-a)(b-y)}}=\frac{\pi}{\sqrt{a b}} \text {, and } \\
& \int_{a}^{b} \frac{d y}{y^{2} \sqrt{(y-a)(b-y)}}=\frac{a+b}{2(a b)^{3 / 2}} \pi .
\end{aligned}
$$

The integration result is

$$
\begin{aligned}
\delta_{0}^{(n)}= & \frac{\pi q^{6}}{8}(n+l+1)^{-2}\left(-\varepsilon_{01}^{(n !}\right)^{-3: 2} \\
& -\frac{\pi q^{2}}{2}(n+l+1)^{-1}(2 n+2 l+1)\left(-\varepsilon_{0 i}^{1 n !}\right)^{-1: 2} \\
& +\pi(n+l)^{1: 2}(n+l+1)^{1: 2} .
\end{aligned}
$$

So the quantization condition Eq. (19) from Eqs. (25) and (28) is, by setting $z=\frac{q^{2}}{2}\left(-\varepsilon_{\mathrm{i}^{1}}^{\mid n i}\right)^{-1: 2}$.

$$
\begin{aligned}
\pi z- & \pi(n+l)^{1: 2}(n+l+1)^{1: z}+\pi(n+l+1)^{-2} z^{3} \\
& -\pi(n+l+1)^{-1}(2 n+2 l+1) z \\
& +\pi(n+l)^{1: 2}(n+l+1)^{1: 2}=\pi .
\end{aligned}
$$

Rearranging it. we obtain the cubic equation to solve.

$$
z^{3}-(n+l)(n+l+1) z-(n+l+1)^{2}=0 .
$$

It has three solutions. i.e.

$z=-(n+l+1) / 2 \pm \sqrt{(n+l+1)(n+l-3)} / 2$ and $z=n+l$ +1 , but only the positive solution $(z=n+l+1)$ is meaningful. Therefore.

$$
n+l+1=\frac{q^{2}}{2}\left(-\varepsilon_{0}^{1 n i}\right)^{-1 ;} .
$$

We funally obtain $\varepsilon_{0}^{(n)}=-\frac{q^{+}}{4(n+l+1)^{2}}$ that is identical with the eigenenergy given in $\mathrm{Eq}$. (24). In conclusion the ATMM quantization condition produces the eigenenergy of the coulomb potential easily when it is combined with supersymmetry algebra.

Now let us determine the scattering-led phase shift $\delta_{r}$. As seen in Eq. (28). $\delta_{i 1}^{\prime \prime \prime}$ of the $n$-th partner Hamiltonian depends on the eigenenergy $\varepsilon_{n}\left(=\varepsilon_{i}^{(n)}\right)$. Inserting the eigenenergy' of Eq. (24) into Eq. (28), it has a simple form. i.e..

$$
\delta_{0}^{(n)}=\pi \sqrt{(n+l)(n+l+1)}-\pi(n+l)
$$

Comparing Eq. (5) with Eq. (22), one finds that $V^{(m)}(r)$ can be obtained by replacing $/$ with $n+l$ in $V(r)\left(=I^{(0)}(r)\right)$. One can evaluate $\dot{P}_{0}(r)$, i.e..

$$
\begin{aligned}
P_{i 1}(r) & =-\frac{d \Psi_{0}(r) / d r}{\Psi_{i 1}(r)}=-\frac{d\left\{r R_{1 l}(r)\right\} / d r}{r R_{1 j}(r)} \\
& =\frac{q^{2}}{2(l+1)}-\frac{l+1}{r} .
\end{aligned}
$$

The radial function of the Coulomb potential $R_{13}(r)$ can be found in quantum chemistry textbooks. ${ }^{13}$ Comparing the above Eq. (33) with Eq. (23), it is found $W^{\prime n i}(r)$ can be obtained by replacing $l$ with $n+l$ in $P_{0}(r)$. Recall that $P_{00}(r)=W^{(0)}(r)$. This is a characteristic of the so-called translational shape invariant potentials and, of course. the Coulomb potential is one of them. ${ }^{12}$ Therefore one can conclude that the scattering-led phase shift ${ }^{\mathrm{l}}$ of the Coulomb potential $\delta_{n}$ can be obtained by simply replacing $n+/$ with $l$ in $\delta_{0}^{n !}$ in Eq. (32), i.e.

$$
\delta_{n}=[\sqrt{l(l+1)}-l] \pi .
$$

Generally it is believed that $\delta_{n}$ depends on the quantum number $n$. Our numerical work on a distorted Morse potential confirmed that $\delta_{i}$ is indeed $n$-dependent. ${ }^{19}$ However, for the Coulomb potential, we find in $\mathrm{Eq}$. (34) that $\delta_{n}$ is independent of $n$. i.e.. the scattering-led phase shift is constant for all $n$ states. So far it las been known that $\delta_{n}$ for the harmonic oscillator potential and the Morse potential have a constant $\delta_{n}$ of $1 / 2 \pi{ }^{5,9}$ The reason behind this phenomenon is not understood yet. Furthermore our preliminary work shows that all the shape invariant potentials have a constant $\delta_{n}{ }^{3 i 1}$

\section{Conclusion and Discussion}

Combining the analytical transfer matrix method. Eq. (16) with supersymmetry algebra. a new and exact quantization condition. Eq. (19) is suggested. In this new quantization only the ground state eigenfunctions of supersymmetric partner Hamiltonians are necessary while the original quantization condition requires the eigenfunctions of all states. It reduces mathematical or computational efforts a lot when the eigenenergies of a system are evaluated.

The usage of the new quantization is successfully demon- 

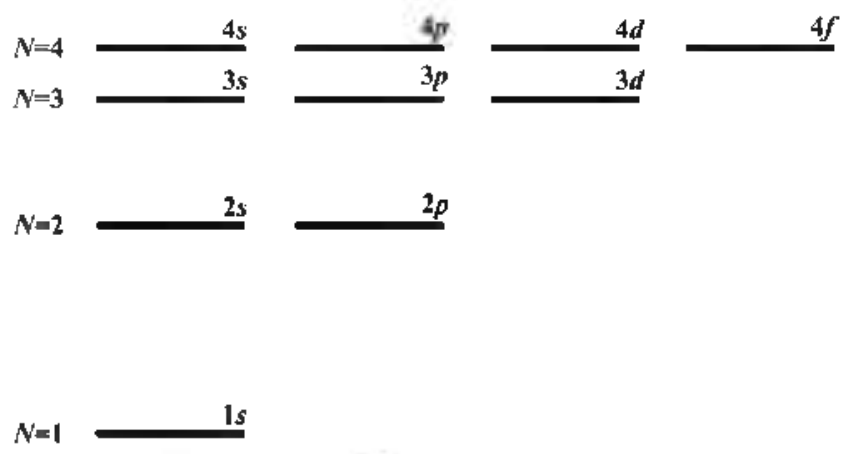

$t=0 \quad t=1 \quad t=2 \quad t=3$

Figure 2. Supersymunetric nature of hydrogenlike atom energy levels

strated for determining the eigenenergies of the Coulomb potential. During the course of work the so-called scatteringled phase shift is evaluated and analyzed. Interestingly the phase shift for the Coulomb potential is found to be constant. i.e. all the eigenstates have the same phase shift for a given 1. Particularly when $l=0$ ( $s$ waves), $\delta_{s}=0$. i.e., there is no phase shift. Then the quantization condition is reduced to a simple form of

$$
\int_{r_{L}}^{r_{R}} \kappa_{n}(r) d r=(n+1) \pi \quad n=0,1.2, \ldots
$$

The above equation i.e., integration of momentum over position variable, is a well-known form of quantization.

For example, the WKB quantization is

$$
\int_{r}^{R} \kappa_{n}(r) d r=\left(n+\frac{1}{2}\right) \pi \quad n=0.1,2 \ldots
$$

As mentioned above the harmonic oscillator and the Morse potentials have $\delta_{n}=1 / 2 \pi$. Inserting it into Eq. (16). one immediately finds that the ATMM quantization is reduced to the above WKB quantization Eq. (36). This is the reason why the WKB quantization is exact for the two potentials 515.21 .2 Therefore $\delta_{n}$ is critical in judging whether a certain quantization condition is exact or not.

Finally we would like to mention more about the supersymmetry of hydrogenlike atoms. From Eq. (24), one immediately finds that the principal quantum number $N(=n+l+1)$ and the angular momentum quantum number $l(=0,1,2 \ldots . N-1)$ are related to each other. For example. for $N=1$. there is only one choice of $n$ and /.i.e. ( $n=0$ and $l=0: 1 s$ wave or orbital). And both have the same eigenenergy because of the same $N$. But for $N=2$, there aret two possibilities (doubly degenerate), i.e. $(n=1$ and $l=0: 2 s$ orbital) and ( $n=0$ and $l=1: 2 p$ orbital). For $N=3$. there exist three degenerate states. i.e. ( $n=2$ and $/=0: 3 s$ orbital). ( $n=1$ and $l=1: 3 p$ orbital). and ( $n=0$ and $l=2: 3 d$ orbital). This relation is illustrated in Figure 2. Comparing Figure 2 with Figure 1, one can understand that hydrogenlike atoms indeed have a superșymmetric structure ${ }^{2 \geq, 24}$

\section{References}

1. Ou. Y. C.: Cao. Z.: Shen. Q. J. Chem. Phys 2004. 121.8175.

2. Froman. N.: Froman. P. O. JWKB Approxintation: Noth Holland: Amsterdam. 1965.

3. Friedrich. H.: Trost. J. Phns. Rep. 2004. 397.359.

4. Cao. Z: Jiang. Y.: Shen, Q.: Dou, X.: Chen, Y. J. Opt. Soc. Am A 1999. 16, 2209

5. Cao. Z.: Liu. Q; Shen, Q; Dou, X: Chen, Y.: Ozaki, Y. Ploss Rev A 2001.63.054103

6. Ou. Y. C.: Cao. Z.: Shen. Q. Phus Lent A 2003. 318.36

7. Zhou. F.: Cao. Z.: Shen. Q. Plns. Rev . 2003.67 .062112

8. He. Y.: Cao. Z; Shen, Q. Phys. Lett \& 2004, 326, 315

9. Sun. H. Pln's Latt. A 2005. 338.309.

10. Bijker. R.: Castanos. O.: Urrutia. L.: Fernandez. D.: Morales-Tecoti, $\mathrm{H}$; Villarreal. C. Supersymmetries in Physics and Its Applications (.4IP Conf. Proc. Iol. 741): Springer-Verlag: New York. 2005.

11. Cooper. F.: Khare. A.: Sukhatme. U. P. Superstnmemy in Ouontum Mechanics: World Scientific: Singapore. 2001.

12. Cooper. F.: Khare. A.: Sukhatme. U. P. Phys. Rep. 1995. 251.267.

13. Levine. I. N. Qtamtum Chemisty, 5th ed.: Prentice Hall: Upper Saddle River. New Jersey, 2000.

14. Cheriaghlou. A.: Fakhri. H. Int. J. Onontm Chent 2005. 101. 291

15. Hruškia. M.: Keung. W.-Y: Sukhatme. U. Phus. Rev .A 1997. 55. 3345 .

16. Sun. H. Bull. Kowan Chem. Soc. 2006. 27. 515.

17. Kostelecky. V. A.: Nieto. M. M. Phns. Rev: Letf. 1984. 53.2285

18. Gradshteyn. I. S.: Ryzhik. I. M. Tables of hntegrals, Series, and Products: Academic Press: New York. 1965.

19. Sur1. H. Bull. Korean Chen. Soc. 2005. 26. 1717.

20. Sun. H. to be published.

21. Jia C.-S.; Wang, J.-Y; He. S.: Sun, L.-T. J. Phys. A. Math. Gen 2000. 33,5045 .

22. Ivanov. I. A. J. Phws A: Math. Gen. 1997. 30.3977.

23. Haymaker. R. W. Rau. A. R. P. Ant J. Phys. 1986. 5 t. 928.

24. Lahiri. A.: Roy. P. K.: Bagehi. B. Int. J. Mod Phys. 1990. 5.1383. 